Rev. Elev. Méd. Vét. Pays trop. 1969. 22, 3 (337-355).

\title{
Enquête sur la tuberculose bovine au moyen de tests tuberculiniques dans diverses régions d'Afrique occidentale (Haute Volta et Côte d'Ivoire) \\ Résultats et considérations générales
}

\author{
por R. GIDEL, J. P. ALBERT ef M. RETIF \\ Avec la collaboration technique de A. CISSE ef M. SIMPORE
}

\begin{abstract}
RÉSUMÉ
En 1967 et 1968, les Sous-Sections Tuberculose et Zoonoses du Centre Muraz à Bobo-Dioulasso, ont effectué 5 enquêtes couplées sur l'épidémiologie de la tuberculose humaine et de la tuberculose bovine en Afrique de l'Ovest. Ces enquêtes ont été effectuées au moyen de tests tuberculıniques et par sondage aléatoire dans diverses zones climatiques de Haute-Volfa et de Côte-d'lvoire. allant du quinzıème degré de lahıtude Nord (zone sahélienne) au quatrième degré de latitude Nord (zone côtière).

Les auteurs exposent ici les résultałs de ces enquêtes pour ce qui concerne la tuberculose bovıne. 8.132 bovins ont éré tuberculınés au total; chaque animal qu été l'objet d'une double tuberculınation effectuée sımulianément en deux régions différentes à l'aide de tuberculine humonobovine et tuberculıne avıaire.

II ressort de l'analyse statistique des résultats que des différences significatives sont observées d'une part entre les régıons prospectées el d'autre part, dans une même région, selon l'âge des animaux tuberculinés. Enfin, l'étude comparative des résultats des enquêfes humaines et animales en régı́on sahélienne montre l'incidence de la tuberculose bovine sur la population humaine de ces régions en particulier chez les jeunes enfants.
\end{abstract}

\section{INTRODUCTION}

La tuberculose, Sogo-Sogo Gwé («ia toux blanche ») en langue Bambara, fut, de tous temps, redoutée des populations d'Afrique Noire. Les dix dernières années ont vu les autorités sanitaires délaisser quelque peu cette endémie pour se pencher sur d'autres problèmes estımés plus urgents (onchocercose, rougeole, variole). Or, les dernières publications de l'OMS montrent que la tuberculose, loin d'avoir régressé, a repris son expansion pour se placer à nouveau parmi les endémies majeures les plus préoccupantes.
Par ailleurs, M. G. REGNOULT (1963), à la suite d'une étude bibloggraphıque, avalt attiré l'attention sur la place importante occupée par la tuberculose bovine dans certaınes régions de l'Afrique de l'Ouest. Pour situer l'importance exacte de cette endémie, tant en pathologie humaine qu'en pathologie vétérınaire, Il fallait entreprendre une vaste enquête menée parallèlement chez l'homme et chez l'anımal. Encore fallait-il disposer d'un procédé facilement applicable sur le terrann, quelle que soit la région prospectée, qui permette de comparer les résultats et tirer des conclusions statistiquement 
valables. La méthode d'échantıllonnage aléatoire proposée par LEFEVRE ef GATEFF (1966) semblait convenir parfaitement pour les enquêtes humaines. C'est elle qui fut adoptée pour celles$\mathrm{ci}$, tandis que les enquêtes animales étaient, chaque fors que cela s'avérait possible, menées paralièlement, afin de comparer les résultats ef d'établir, le cas échéant, certaines corrélations entre les deux affections.

Les S/Sections Tuberculose et Zoonoses du Centre Muraz ont donc entrepris, au cours des années 1967 et 1968, une série d'enquêtes par tests tuberculıniques dans différentes zones climatiques de l'Afrique de l'Ovest allant du $15^{\mathrm{e}}$ degré de latitude Nord au 4e degré de latitude Nord (Voir cartes 1 et 2). Cinq régions ont été ainsi prospectées : zone de savane sahélienne (région de Dori en Haute-Volta) ; zone de savane soudanienne (région de Dédougou en HauteVolta) ; zone de savane guinéo-soudanienne (région de Boundiali en Côte-d'lvoire) ; zone de forêt (région de Daloa en Côte-d'lvoire) ; zone côtière (région de Sassandra en Côte-d'Ivoire). Deux équipes travaillant en collaboration avaient été constituées : l'une, dirigée par un médecin, était chargée de l'enquête humaine : l'autre, dirigée par un vétérinaire, était chargée de l'enquête animale.

En ce qui concerne cette dernière, parallèlement à l'enquête tuberculinique, une enquête statistique a été menée auprès des abattoirs des régions prospectées.

Le présent travail expose les résultats obtenus en ce qui concerne la tuberculose bovine. Les résultats intéressant la fuberculose humaine font l'objet d'une autre publication.

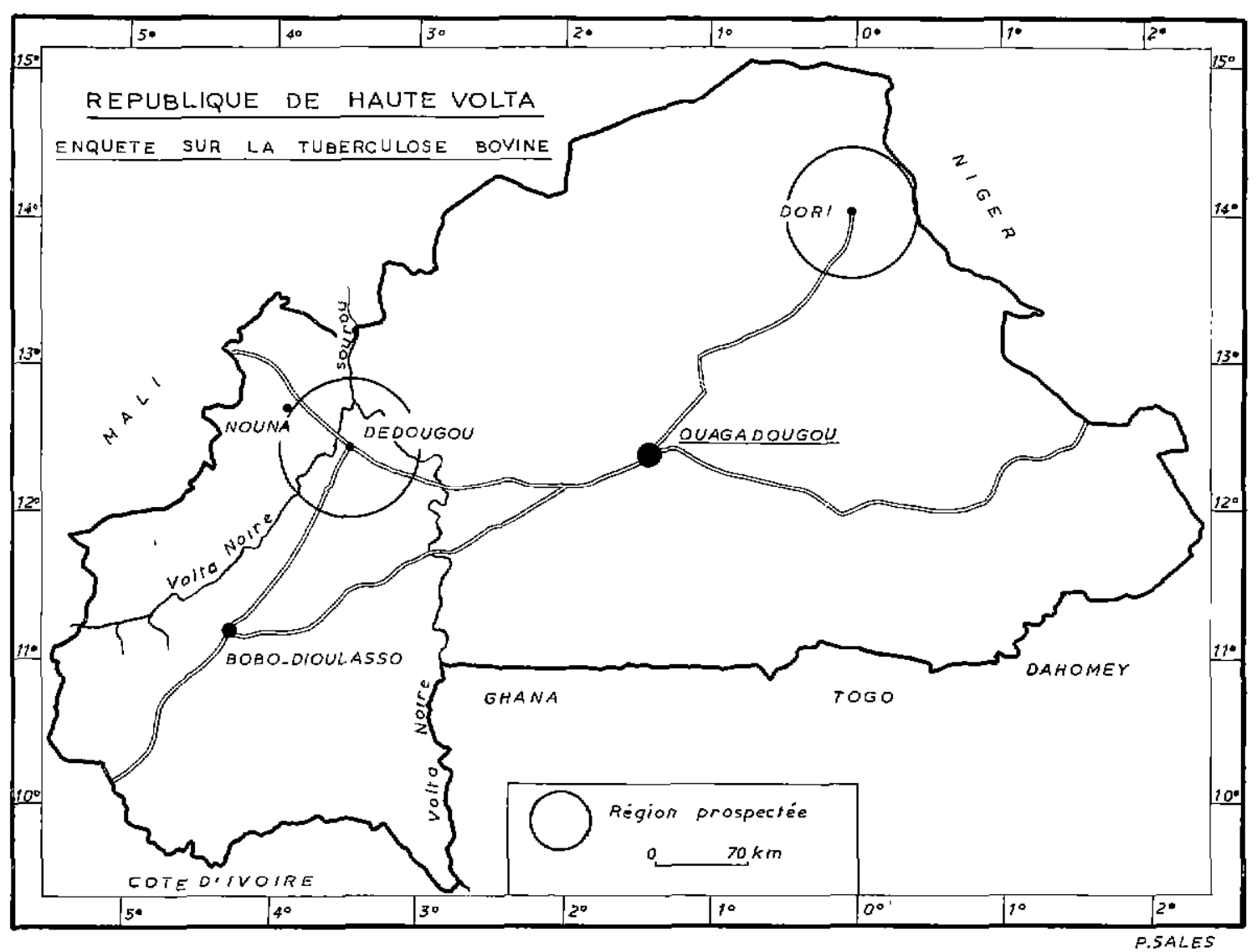

Carte 1، --Situation géographique des régions prospectées en Haute-Volta (région de Dédougou-Nouna et région de Dori). Position de Bobo-Dioulasso par rapport à la Capitale Ouagadougou. 


\section{REPUBLIQUE DE COTE DIVOIRE}

ENQUETE SUR LA TUEERCULOSE BOVINE

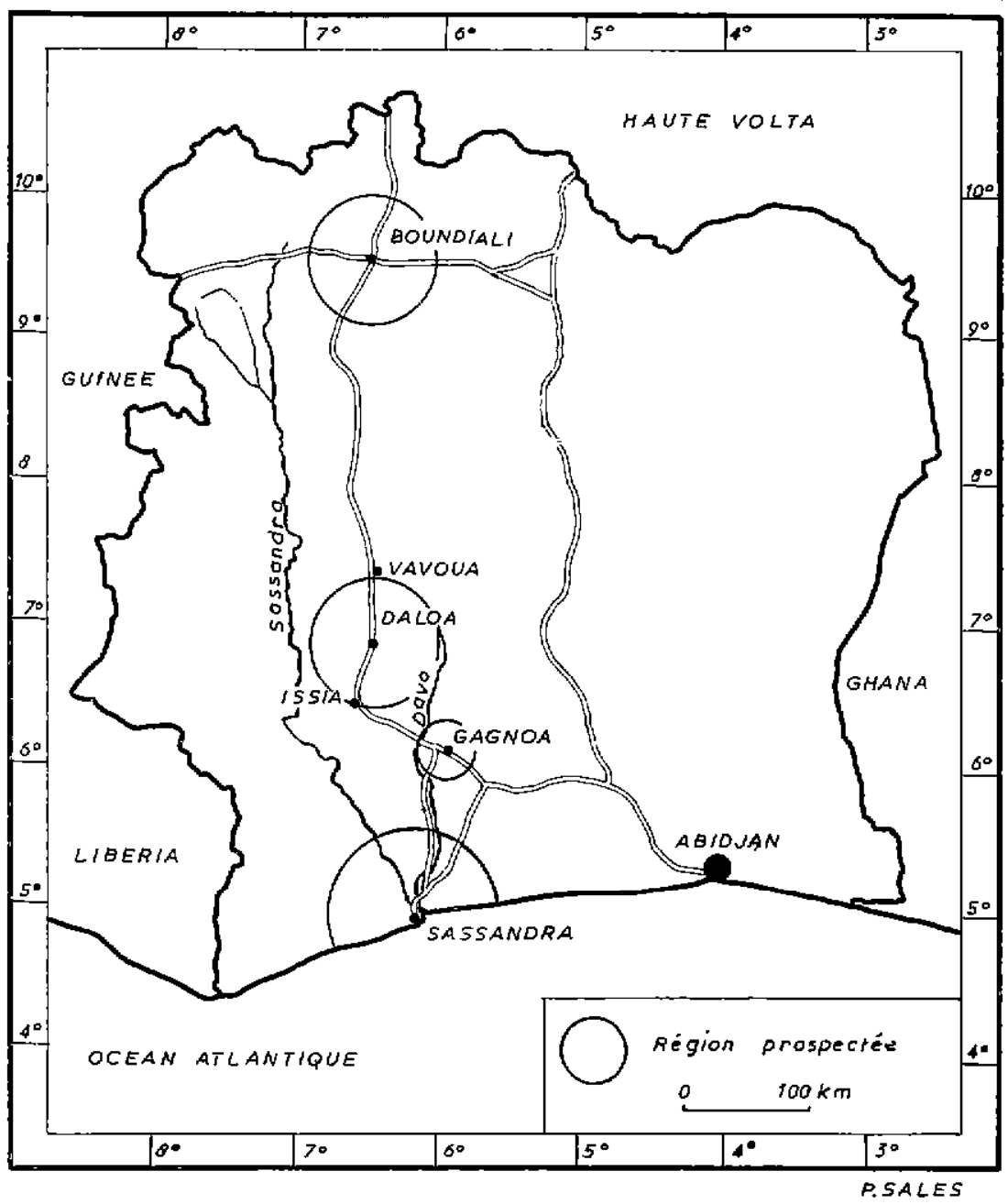

Carte 2. - Situation géographique des régions prospectées en Côte d'Ivoıre (région de Boundiali, de Daloa-Gagnoa ef de Sassandra).

\section{PRÉSENTATION DES RÉGIONS PROSPECTÉES}

\subsection{Région de Dori.}

\subsubsection{Géographie ef climat.}

La région de Dori se trouve située entre les $13^{\mathrm{e}}$ et $15^{\mathrm{e}}$ degrés de latitude Nord et les premiers degrés de longitude Est et Ouest. C'est une zone faiblement vallonnée avec des dunes sablon- neuses alternant avec des bas-fonds argileux et de nombreux affleurements rocheux. La majeure partie de cette région est comprise entre 230 et 350 mètres d'altitude.

Le paysage est celui d'une savane à épineux au Sud ef d'une steppe herbeuse avec un couvert peu dense d'épineux au Nord. II n'y a pas de cours d'eau permanents et la majorité des mares et marécages tarissent au cours de la saison 
sèche. La nappe phréatique est souvent profonde. Le problème de l'abreuvement des troupeaux et celui des pâturages se posent alors de façon aigue. En saison des pluies se forment de nombreux cours d'eau temporares ef tous les basfonds limoneux sont inondés. La circulation est alors difficile pendant plusieurs mois.

Le climat est soudanien avec une seule saison des pluies de maı à septembre. Les précipitations moyennes annuelles sont de 500 à $600 \mathrm{~mm}$ dans les environs de Dorl et sensiblement plus farbles au Nord et plus importantes au Sud. L'amplitude des variations thermiques quotidiennes est considérable, particulièrement durant la saison fraîche (novembre à février). Les écarts entre les maxima et les minima mensuels moyens peuvent alors dépasser $20^{\circ}$. L'humidité relatıve est faible ou très faible durant la majeure partie de l'année.

La population comprend principalement des Sonrals, des Peuhls, des Touaregs et leurs anciens captifs, les Bellas. Les Peulhs et les Touaregs sont nomades et campent à proximité des pâturages de leurs troupecux. Les sédentaires ont des habitations groupées en petits et moyens villages. On trouve souvent, à proxımité de ceux-ci, des enclos en épineux où le bétal est parqué pendant la nuit. Les campements nomades sont assez dispersés. Chez les Peuhls, les cases hémisphériques sont faites de nattes de paille disposées sur une armature de branchages. Chez les Touaregs, les abris sont généralement constitués de peaux tendues sur des armatures de branchages également.

\subsubsection{Ressources économiques.}

Dans cette région, l'agriculture est limitée à quelques cultures vivrières, mil et sorgho surtout. La vocation essentielle de ce pays est l'élevage des grands et petits rumınants. Les effectıfs dénombrés par le service de l'Elevage pour l'ensemble de la circonscription de Dori sont les suivants :

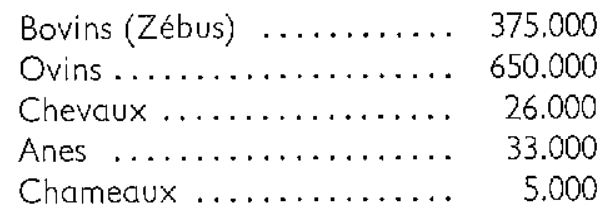

L'aviculture est très répandue dans cette région. Cependant, la découverte d'un important gisement de manganèse aura vraisemblablement des conséquences heu reuses sur le développement de cette région, notamment par suite de la construction d'une voie ferrée qui la reliera au réseau déjà existant «Abidjan-Niger». L'extension de l'élevage est toutefois conditionnée par le problème des pâturages en saison sèche.

\subsection{Région de Dedougou.}

\subsubsection{Géographie et elimat.}

La région prospectée est située entre les $12^{\mathrm{e}}$ et $13^{\mathrm{e}}$ degrés de latitude Nord et les $3^{\mathrm{e}}$ et $4 \mathrm{e}$ degrés de longltude Ouest. C'est une zone de savane arborée très peu vallonnée. Hormis la Volta Norre, II n'y a pas de cours d'eau permanents.

Par contre en saison des pluies apparaissent des cours d'eau temporaires et de nombreuses mares se forment. En salson sèche, l'eau est à une profondeur de 10 mètres environ.

Le climat est de type soudano-guinéen avec une saison des pluies qui s'étend de mai à octobre avec pluviométrı maxima en juillet et doût. La moyenne annuelle se situe aux environs de $1.100 \mathrm{~mm}$.

Des pâturages permanents existent le long de la Volta Noire. Du point de vue population, 4 groupes ethniques se rencontrent dans cette région ; les Bobo-Oulé ef les Bobo-Fing, en général fétichıstes avec cependant une influence marquée du christianisme. C'est une population grégaire, prolifique, très particulariste, groupée en gros villages. Ce sont de bons cultivateurs, mals de mauvais éleveurs. L'élevage est aux mains des Peuhis dont un certain nombre se sont sédentarısés à proximité des points d'eau et pâturages permanents. Ils deviennent alors d'excellents cultivateurs sachant utiliser la fumure animale et confient une partie de leur troupeau aux Peuhls transhumants. Enfin, il convient de signaler dans le Nord-Ouest de cette région, une migration importante depuis quelques années de Mossi originaıres du Yatenga (région Nord de Ouahigouya) à la recherche de nouvelles terres.

\subsubsection{Ressources économiques.}

A côté des cultures vivrières (mil, sorgho, maïs) qui sont en général excédentaires, il faut 
surtout retenir la culture du coton, bien accueillie par les populations et qui, sous l'impulsion de la CFDT, est passée de 1.500 tonnes en 1963 à 10.000 tonnes en 1967. Son expansion continue et Il est prévu un tonnage de 25.000 tonnes en 1972. La culture de l'arachide n'a pas connu le même succès auprès des cultivateurs et reste limitée à la couverture des besoins locaux.

L'élevage est très important dans cette régıon. Les effectifs recensés par le service de l'Elevage sont les suivants :

$\begin{array}{lr}\text { Bovins } \ldots \ldots \ldots \ldots \ldots \ldots \ldots & 300.000 \\ \text { Ovins et caprins } \ldots \ldots \ldots \ldots & 300.000 \\ \text { Porcs } \ldots \ldots \ldots \ldots \ldots \ldots \ldots & 16.000 \\ \text { Chevaux } \ldots \ldots \ldots \ldots \ldots \ldots & 7.000 \\ \text { Anes } \ldots \ldots \ldots \ldots \ldots \ldots & 17.000\end{array}$

L'aviculture est également très développée dans la plupart des villages.

\subsection{Région de Boundiali.}

\subsubsection{Géographie et climat.}

La région prospectée est siłuée entre les ge et $10^{\circ}$ degrés de latitude Nord et les $6^{\mathrm{e}}$ et $7^{\mathrm{e}}$ degrés de longitude Ouest. Elle se présente comme un plateau doucement valionné, d'une altitude moyenne de 350 à 400 mètres, avec quelques collınes, atteignant 700 mètres. L'aspect général est tantôt celui de savane boisée, tantôt celui de forêt claire. Les cours d'eau sont nombreux, mais beaucoup tarissent en totalité au en partie en scison sèche. On y rencontre cependant plusieurs petits cours d'eau permanents bordés por des forêts galeries.

La nappe phréatique n'est jamais profonde et 'les habitants peuvent se procurer de l'eau sans grandes difficultés, soit à l'aide de puits rudimentaires, soit en recreusant le lit des cours d'eau asséchés.

Le climat, de type guinéo-soudanien, est à deux saisons : une saison des plules unique s'étendant de mai à octobre et une saison sèche s'étendant de novembre à avril, maıs surtout très marquée de décembre à fin mars. Les pluies sont variables en volume et en répartition avec un chiffre moyen annuel de $1.500 \mathrm{~mm}$. Plus de $4 / 5$ des pluies tombent de mai à octobre et la pluviométrie est très forte de jull let à septembre avec maximum en août. Les températures minimales moyennes sont comprises entre $17^{\circ}$ (décembre et janvier) et 230 (avril) et les températures maximales moyennes entre $29^{\circ}$ (aoôt) et $36^{\circ}$ (février et mars).

La densité de la population dans la région prospectée est de 10 à 15 habitants au $\mathrm{km}^{2}$. Deux grands groupes ethniques se rencontrent dans cette région : les Malınkés et les Sénoufo. Les Malinkés sont venus du Nord-Ouest et ont repoussé vers l'Est les Sénoufo.

lis sont profondément islamisés et tirent leurs principales ressources des cultures vivrières ef de l'élevage. Les Sénoufo qui, en fait, constituent un complexe ethnique, chaque sous-groupe constıtuant lui-même une ethnie avec ses traditions propres, sont d'excellents cultivateurs. Bien que I'Islam semble en faveur dans cette région depuis une douzaine d'années, beaucoup de villages Sénoufo ont encore conservé leurs structures sociologiques et traditionnelles ef leurs rites coutumiers.

\subsubsection{Ressources économiques.}

Jusqu'à ces dernières années, la régıon était surtout vouée aux cultures vivrières : igname (qui tient une part importante dans l'alımentation), riz (qui tient la deuxième place dans l'alimentation), mass, sorgho, mil, arachıde. Toutefois, la culture du coton démarrée en 1960 a connu depuis un développement spectaculaire par l'édification en 1967 sous. l'égide de la CFDT d'une usıne d'égrenage à Boundiali, capable de traiter annuellement 12.000 tonnes de cotongraine.

Malgré le développement de l'Agriculture, il demeure de vastes possibilités en matière d'élevage. 70.000 bovins, tous des taurins, ont été recensés dans le secteur vétérınaire de Boubdialı. Nous avons été frappés par l'état d'entretien remarquable dans lequel se trouvaient tous les anımaux que nous avons approchés au cours de cette enquête, alors que nous étions en fin de saison sèche. Eu égard aux nombreux pâturages existants et à l'absence de véritables problèmes d'abreuvement, il est bien certain que cet élevage est susceptible de connaitre encore un large développement, dans la mesure évidemment où des prix suffisamment rémunérateurs seront offerts aux éleveurs. L'élevage des volaılles est également plus ou moins répandu dans la plupart des villages que nous avons prospectés. 


\subsection{Régions de Daloa ef Gagnoa.}

\subsubsection{Géographie et climaf.}

Les régions prospectées sont situées entre les $5^{\mathrm{e}}$ et $8^{\mathrm{e}}$ degrés de latitude Nord et les $5^{\mathrm{e}}$ et $7 \mathrm{e}$ degrés de longitude Ouest. Ce sont des zones de forêt dense, plates, sans relief. La saison des pluies s'étend de mars à octobre ef la pluviométrie annuelle moyenne est de $1.500 \mathrm{~mm}$ environ. Le degré hygrométrique est toujours élevé ef de ce fait les quelques pâturages sont toujours verdoyants. Les températures maximales moyennes ef minimales moyennes sont comprises entre 33 et $21^{\circ}$ centigrades.

\subsubsection{Ressources économiques.}

L'économie de ces régions est dominée par l'exploitation de la forêt. On y trouve cependant quelques cultures vivrières, bananes, café et cacao, et quelques ruminants. Il est possible d'envisager une extension substantielle de cet élevage et les autorités responsables consacrent des moyens importants à cet effet en incitant et en favorisant la création de noyaux d'élevage.

\subsection{Région de Sassandra.}

\subsubsection{Géographie ef climat.}

La région prospectée est située en zone côtière entre les $4 \mathrm{e}$ et $6^{\mathrm{e}}$ degrés de latitude Nord ef les $6^{\mathrm{e}}$ e $^{\dagger} 7^{\mathrm{\theta}}$ degrés de longitude Ouest. C'est une zone de collines, initialement couverte de forêts mais dont l'aspect a été considérablement modifié par l'exploitation du bois et l'agriculture. La côte est bordée de petites plages à cocotıers avec des villages de pêcheurs. Il y a de nombreux cours d'eau permanents dans la régıon, les plus importants étant le fleuve Sassandra et le Davo. Lo plus grande partie du pays est à moins de 100 mètres d'altitude avec quelques zones situées entre 100 et 200 mètres.

Du point de vue climatique, on distingue deux saisons des pluies. L'une s'étend de mars à juillet (grande saison des pluies) et l'autre d'octobre à décembre (petite saison des pluies). La pluviométrie varie de 1.411 à $1.800 \mathrm{~mm}$ avec une pluviométrie moyenne de $1.600 \mathrm{~mm}$.

La répartition mensuelle est sujette à des variations assez importantes selon les années, mai à juın étant néanmoins les mois à précipita- tıon maximale. La saison sèche peut être très marquée certaines années. Les températures minimales moyennes sont comprises entre $21^{\circ}$ et $23^{\circ}$ et les maximales moyennes entre $26^{\circ}$ et $30^{\circ}$.

\subsubsection{Ressources économiques.}

La zone littorale proprement dite comprend de vastes plantations de palmıers à huile. A l'intérieur, à côté de l'exploitation traditionnelle des bois précieux amenant une disparition ou une dégradation de la forêt, on rencontre des plantations de café, des plantations de bananes en régression, des plantations d'agrumes en pleine expansion. Ces dernières sont orientées essentiellement vers la production d'essences de citron et de bergamote. A ces cultures s'ajoutent, bien entendu, les cultures vivrières traditionnelles.

L'élevage des petits ruminants (moutons essentiellement) des porcs ef des volailles se rencontre à peu près dans tous les villages. Par contre l'élevage du gros bétail est beaucoup plus limité. Il est cantonné à un certain nombre d'exploitations agricoles qui entretiennent un troupeau de bovins essentiellement en vue de la production de fumier indispensable à leurs cultures. Néanmoins ces exploitants s'intéressent pour la plupart à leur troupeau et s'efforcent d'en améliorer les possibilités zootechniques. par des croisements appropriés. On rencontre dans cette région la race des lagunes, des N'Damas, des métis N'Damas-lagunes, des métis N'Damas-lagunes-Baoulés et des métis N'Damas-jerseys. Les animaux sont en général remarquablement entretenus et il ne semble pas. y avar de problème pathologique majeur. Ceci démontre les possibilités latentes de cette région au point de vue élevage. Si celui-ci restera toujours un élément d'appoint, il mérite néanmoins d'être encouragé et développé, eu égard aux possibilités de la région et aux perspectives offertes par les croisements JerseysN'Damas. II serait toutefois indispensable que des prix plus rémunérateurs soient proposés aux producteurs, étant donné la qualité des animaux.

\section{MÉTHODE D'ENQUÊTE ET TECHNIQUE}

L'enquête animale a été menée parallèlement à l'enquête humaine lorsque cela s'est avéré 
possible. Selon la densité du cheptel dans la région prospectée, elle a été réalisée solt de façon exhaustive, solt par sondage aléatore. Dans ce dernier cas, 20 villages de type sédentaire étaient choisis par tirage au sort, tant pour l'enquête humaine que pour l'enquête animale, ces villages étant les mêmes pour les deux enquêtes, afin de pouvoir éventuellement faire certaines études comparatıves. Dans chaque village retenu, tout le cheptel qui a pu être tuberculiné l'a été, y compris les jeunes animaux.

Les séances de fuberculinations étaient arganisées de la façon suivante : les chefs des villages intéressés étaient informés en temps utile, à la fois par l'administration générale et par les agents locaux responsables ou service de l'élevage, des jours exacts de ces séances et étaient invités à faire rassembler tout le cheptel sédentaire du village. Lorsqu'il existait un parc à vaccination en béton à proxımité de celui-ci, les animaux devant être tuberculinés y étaient menés. Dans le cas contraire, les animaux étaient parqués dans l'enclos en épıneux ou en bois du village, si celui-cl en disposait. Lorsqu'aucune de ces deux solutions n'était possible, les bergers construisaient un parc sommaire en épineux ou en autre bois selon la région.

Suivant les recommandations de A. LUCAS (1967 ef 1968), nous avons utılisé la tuberculination intradermique comparative. Chaque animal a donc été l'objet d'une double tuberculination effectuée sımultanément en deux points séparés avec deux tuberculines différentes : tuberculine synthétique humano-bovine et tuberculine aviaire, toutes deux de l'Institut Mérieux. Les injections intradermiques ont été pratiquées à l'aide de seringues HAUPTNER à carpules interchangeables pour tuberculinations en série. Ce type de seringues et le système des carpules se sont révélés extrêmement pratiques vu les conditions de travail particulières et, en tous cas supérieurs aux autres techniques que nous avons expérimentêes. Nous avons d'abord essayé les tuberculinations au niveau de l'encolure mais nous les avons abandonnées pour des raisons pratiques (difficultés de contention et nécessité de couper les poils sur les races locales). Nous avons finalement donné notre préférence aux tuberculinations intradermiques au niveau des deux plis caudaux qui, étant donné les conditions de travail en milieu tropical, nous permettaient de tuberculiner beaucoup plus d'anımaux dans le même laps de temps, et dont les résultats sont aussi valables que ceux obtenus à l'encolure (A. LUCAS, 1967). Chaque animal, quel que soit son âge, a reçu $1 / 10$ de $\mathrm{ml}$ de chacune des deux tuberculines dans le derme de chaque pli caudal (soit 2.500 unités) : pli caudal droit pour la tuberculine humano-bovine, pli caudal gauche pour la tuberculine aviaire. Tout animal tuberculiné était aussitôt marqué au mercurochrome afin de pouvoir l'identifier au moment du contrôle. II arrive en effet presque chaque fois que quelques animaux s'échappent du parc au cours des séances de tuberculination. II convient également que les animaux tuberculinés puissent être distingués des anımaux appartenant d̀ des troupeaux des villages voisins, auxquels ils pourraient parfois se mélanger. Signalons toutefois que si la solution qui consiste à utiliser le marquage au mercurochrome est facile et nous a donné en général satisfaction, il nous. paraît préférable d'utiliser une peinture indélébile qui ne soit pas effacée en cas de pluies ou même simplement au contact des herbes en climat humide.

Une tornade survenue entre les séances de fuberculination et de contrôle peut en effet effacer la marque au mercurochrome de certains animaux.

Les lectures ont été faites en général 72 heures plus tard. Toutefois, dans quelques cas particuliers et pour des raisons impératives (problèmes de véhicules par exemple), certains contrôles ont été faits entre 48 et 72 heures. Au moment du contrôle, on notalt, en même temps que les résultats, l'âge et le sexe des animaux, et ceux-ci étaient marqués au bleu de méthylène. Là encore il peut arriver que quelques animaux échappent au contrôle, n'ayant pu être amenés par leurs propriétalres. Ceux qui s'échappent parfois du parc au cours du contrôle sont en général capturés à nouveau pour ne pas perdre le bénéfice de l'examen, mais cecl occasionne des perfes de femps importantes.

Des difficultés de tout ordre n'ont pas manqué de surgir au cours de ces enquêtes, plus particulièrement dans les régions sahéliennes (diffıcultés pour rejondre certains villages choisis par le tirage au sort, plusieurs heures étant parfois 
nécessaires du fait des pistes en mauvais état ou même parfois inexistantes ; difficultés de langue ef d'orientation dans certaınes régions d'où nécessité d'avoir un guide servant en même temps d'interprète; pannes de véhicules, pannes des groupes électrogènes qui alimentaient les réfrigérateurs où était slockée la tuberculıne pour les deux enquêtes humaine et animale, parcs à vaccinations mal conçus ou en mauvais état. Ces enquêtes ont pu néanmoins être menées à bien grâce au concours très efficace que nous ont apporté les Services de l'Elevage des régions intéressées en mettant à notre disposition soit du personnel connaissant parfaitement la région et les éleveurs, soit du matériel : véhicules, réfrıgérateurs, congélateurs, fourniture de glace.

Enfin, comme il a été dit précédemment, une enquête statistique a été menée auprès des abattoirs des régions prospectées afin de noter le nombre d'animaux reconnus tuberculeux à l'inspection des viandes au cours des dernières années.

\section{RÉSULTATS DES TUBERCULINATIONS}

\subsection{Résultats d'ensemble.}

Le tablecu 1 indıque le nombre de bovins tuberculinés par rapport au nombre d'animaux contrôle pour des raisons diverses évoquées. plus haut.

Au cours des 5 enquêtes, 8.132 animaux au total ont été tuberculinés et 7.536 ont été contrôlés, soit un pourcentage de 92,67 p. 100.

Les résultats globaux des tuberculinations ont montré que 440 bovins, soit 5,84 p. 100 des animaux contrôlés, ont présenté une réaction à l'une ou à l'autre des 2 tuberculines, tandis. que 42 , solt 0,56 p. 100 ont présenté une réaction à la fois vis-à-vis de la tuberculine humanobovine ef de la tuberculine aviaire. En ce qui concerne ces 42 animaux, nous avons considéré que lorsque la réaction à la tuberculine aviare étaif supérieure à la réaction à la tuberculine. humano-bovıne, il s'agissait d'une sensibilisation liée à l'action de M. avium ou d'une mycobactérie atypique (LUCAS 1968). 9 anımaux ont présenté une réaction de ce type et ont donc été considérés comme sensibilısés par $M$. avium ou une mycobactérie atypique, tandis que les 33 autres ayant présenté une réaction plus forte ou égale avec la tuberculine humano-bovine ont été considérés. comme ayant été sensibilisés par $M$. bovis (ou M. tuberculosis). Pour l'expression des résultats, nous avons donc classé dans le tableau $2 \operatorname{ces} 9$ et 33 anımaux respectivement parmi ceux positifs à la tuberculine aviaıre ou parmi ceux positifs à la tuberculine humano-bovine.

TABLEAU 1

Nombre de bovins recensés, tuberculinés et contrôlếs dans les cinq rëgions prospectêes

\begin{tabular}{|c|c|c|c|c|}
\hline \multirow{2}{*}{ Régions prospectëes } & \multicolumn{3}{|c|}{ Nambre de bovins } & \multirow{2}{*}{$\begin{array}{c}\text { Bovins contrôlës par rapport } \\
\text { aux bovins tuberculinés } \\
\text { (p.100) }\end{array}$} \\
\hline & Recensës & Tuberculinës & Contrôlés & \\
\hline DORI (Haute-Volta) & 156.791 & 2.361 & 2.231 & 94,49 \\
\hline DEDOUGOU (Haute-VoIta) & 172.550 & 2.698 & 2.432 & 90,14 \\
\hline BOUNDIALI (Côte d'Ivoire) & 70.000 & 1.844 & 1.683 & 91,27 \\
\hline DALOA-GAGNOA (Côte d'Ivoire) & 2.086 & 652 & 613 & 94,00 \\
\hline SASSANDRA (Côte d'Ivoire) & 577 & 577 & 577 & 100,00 \\
\hline Total & 402.004 & 8.132 & 7.536 & 92,67 \\
\hline
\end{tabular}

recensés dans les différentes régions prospectées, ainsi que le nombre d'animaux contrôlés par rapport aux animaux tuberculinés. En effet, comme nous l'avons précédemment rapporté, quelques animaux échappent en général au
Le tableau 2 indique les résultats obtenus avec chacune des deux tuberculines dans les différentes régions prospectées. L'étude statıstique des résultats obtenus avec la tuberculine humanobovine montre qu'une différence hautement 
TABLEAU 2

Résultats des tuberculinations par tuberculine et par rëgion

\begin{tabular}{|c|c|c|c|c|c|c|c|}
\hline \multirow[b]{2}{*}{$\begin{array}{l}\text { Régions } \\
\text { prospectếes }\end{array}$} & \multirow[b]{2}{*}{$\begin{array}{l}\text { Nombre d'animaux } \\
\text { tuberculinés et } \\
\text { contrôlês }\end{array}$} & \multicolumn{3}{|c|}{$\begin{array}{c}\text { Animaux positifs à la tuberculine } \\
\text { humano-bovine }\end{array}$} & \multicolumn{3}{|c|}{$\begin{array}{c}\text { Animaux positifs à la tuberculine } \\
\text { aviaire }\end{array}$} \\
\hline & & Nombre & Pourcentage & $\begin{array}{l}\text { Intervalle de } \\
\text { confiance } \\
\text { avec risque } \\
5 \text { p. } 100\end{array}$ & Nombre & Pourcentage & $\begin{array}{l}\text { Intervalle de } \\
\text { confiance } \\
\text { avec risque } \\
5 \mathrm{p} .100\end{array}$ \\
\hline DORI & 2.231 & 133 & 5,96 & $4,98-6,94$ & 21 & 0,94 & $0,00-2,21$ \\
\hline DEDOUGOU & 2.432 & 69 & 2,84 & $2,18-3,50$ & 94 & 3,86 & $3,10-4,62$ \\
\hline BOUNDIALI & 1.683 & 52 & 3,09 & $2,28-3,90$ & 58 & 3,45 & $2,59-4,31$ \\
\hline DALOA-GAGNOA & 613 & 4 & 0,65 & $0,18-1,70$ & 0 & 0,00 & 0 \\
\hline SASSANDRA & 577 & 0 & 0,00 & 0 & 9 & 1,56 & $0,55-2,57$ \\
\hline Total & 7.536 & $25 B$ & 3,43 & $3,01-3,83$ & 182 & 2,41 & $2,02-2,76$ \\
\hline
\end{tabular}

significative existe entre les différentes régions prospectées (chi $2=81,81$ pour 4 degrés de lıberté et $P<10^{-9}$ ). La région la plus infectée est celle de Dorr où près de 6 p. 100 des animaux tuberculınés ont présenté une réaction positive, tandis qu'aucun animal n'a réagi dans la région de Sassandra. II existe également une différence hautement significative pour les résultats obtenus avec la tuberculine aviaire (chi $2=65,40$ pour 4 degrés de lıberté et $\mathrm{P}<10^{-9}$ ). Ici c'est la région de Dédougou qui a présenté le plus de réactions positives $(3,86 \mathrm{p}, 100)$, tandis qu'aucune réaction n'a été notée dans la région de Daloa-Gagnoa.
Nous allons donc étudier les résultats des tuberculinations dans les 5 régions prospectées.

\subsection{Résultats par régions.}

Pour l'expression des résultats, et afin d'étudier le rôle éventuel de l'âge, nous avons, dans chaque région, classé les anımaux en 4 groupes en fonction de celui-ci :

groupe 1 : animaux de 0 à 5 mois groupe II : animaux de 6 à 23 mois groupe III : animaux de 2 à 5 ans groupe IV: animaux de plus de 5 ans.

TABLEAU 3

Résultats par groupe d'âge et par tuberculine pour la région de Dori

\begin{tabular}{|c|c|c|c|c|}
\hline \multirow[b]{2}{*}{ Groupes d'âge } & \multicolumn{4}{|c|}{ Nombre d'animaux } \\
\hline & $\begin{array}{l}\text { Tuberculinés } \\
\text { et contrôlês }\end{array}$ & $\begin{array}{c}\text { Négatifs aux deux } \\
\text { tuberculines }\end{array}$ & $\begin{array}{l}\text { Positifs à la } \\
\text { tuberculine } \\
\text { humano-bovine }\end{array}$ & $\begin{array}{l}\text { Positifs à 1a } \\
\text { tuberculine } \\
\text { aviaire }\end{array}$ \\
\hline Groupe I & 31 & $(70,98 \mathrm{p} \cdot 100)$ & $\left(12,90^{4} \mathrm{p} \cdot 100\right)$ & $\left(16,12^{5} \mathrm{p} \cdot 100\right)$ \\
\hline Groupe II & 676 & $\begin{array}{l}645 \\
(95,42 \mathrm{p} \cdot 100)\end{array}$ & $(2,81 \mathrm{p} \cdot 100)$ & $\left(1,77^{12} \mathrm{p} \cdot 100\right)$ \\
\hline Groupe III & 617 & $\begin{array}{c}586 \\
(94,98 \text { p. } 100)\end{array}$ & $\begin{array}{l}29 \\
(4,70 \mathrm{p} \cdot 100)\end{array}$ & $\left(0,32^{2} \mathrm{p} \cdot 100\right)$ \\
\hline Groupe IV & 907 & $\begin{array}{l}824 \\
(90,85 \mathrm{p} \cdot 100)\end{array}$ & $\begin{array}{c}81 \\
(8,93 \mathrm{p} .100)\end{array}$ & $\left(0,22^{2} \mathrm{p} \cdot 100\right)$ \\
\hline Total & 2.231 & $\begin{array}{l}2.077 \\
(93,10 \mathrm{p} \cdot 100)\end{array}$ & $\begin{array}{l}133 \\
(5,96 \mathrm{p} \cdot 100)\end{array}$ & $(0,94 \mathrm{p}, 100)$ \\
\hline
\end{tabular}




\subsubsection{Région de Dori.}

Près de 6 p. 100 des animaux ont réagi positivement avec la tuberculine humano-bovine tandis que moins de 1 p. 100 ont réagi avec la tuberculine aviaire.

Les résultats par groupe d'âge sont indiqués dans le tableau 3. L'étude statistique des résultats montre qu'une différence hautement significative existe entre les différents groupes, tant pour la tuberculine humano-bovine (ch1 $2=43,82$ pour 3 degrés de liberté et $P<0,00001)$ que pour la tuberculine aviaire (chi $2=19,73$ pour 3 degrés de liberté et $P<0,00001)$. En ce qui concerne la première, les taux les plus élevés ont été rencontrés chez les plus jeunes animaux (12,90 p. 100) et chez ceux âgés de plus de 5 ans ( 8,93 p. 100). Entre ces deux extrêmes, on observe une croissance du taux d'infection en fonction de l'âge. En ce qui concerne la seconde, le taux le plus élevé est rencontré chez les animaux les plus jeunes $(16,12$ p. 100) et les taux les plus faibles chez les animaux âgés de 2 ans ou plus (groupe III : 0,32 p. 100 ; groupe IV : 0,22 p. 100).

$\mathrm{SI}$ l'on considère maintenant les résultats obtenus dans les différents villages, dont nous ne reproduisons pas le détail afin de ne pas surcharger l'exposé, on constate que des variations très importantes peuvent exister d'un village à l'autre en ce qui concerne les réactions à la tuberculine humano-bovine (Kampiti : un animal positif sur 134 , soit 0,7 p. 100 ; Malbo-Kouri :
46 animaux positifs sur 329 soit 14 p. 100). Dans les villages les plus infectés, nous avons rencontré jusqu'à 25 p. 100 d'animaux positifs dans certains troupeaux, cette proportion étant encore plus élevée si l'on considère seulement les animaux les plus âgés.

La tuberculose bovine est donc un problème important pour cette région et nous en étudierons les conséquences dans le chapitre consacré à la discussion des résultats.

\subsubsection{Région de Dedougou.}

2,84 p. 100 des animaux tuberculinés ont réagi positivement à la tuberculine humano-bovine et 3,86 p. 100 à la tuberculine aviaire.

Les résultats par groupe d'âge sont indiqués dans le tableau 4. Malgré les apparences le test statistique ne montre pas de différence sıgnificative en ce qui concerne les résultats obtenus avec la tuberculine humano-bovine (chi $2=4,970$ pour 3 degrés de liberté). Ceci est peut-être dû ¿̀ une insuffisance de l'effectif calculé dans le groupe l. Les résultats obtenus avec la tuberculine aviaire ne montrent également pas de différence significative en fonction de l'äge (chi $2=5,571$ pour 3 degrés de liberté).

Par contre en ce qui concerne cette région de Dédougou, on peut y distinguer, du point de vue géographique, 2 secteurs différents : le secteur de Dédougou proprement dit et le secteur de Nouna qui est en contact avec le Mali et où

TABLEAU 4

Résultats par groupe d'âge et par tuberculine pour la région de Dêdougou

\begin{tabular}{|c|c|c|c|c|}
\hline \multirow{2}{*}{ Groupes d'âge } & \multicolumn{4}{|c|}{ Nombre d'animaux } \\
\hline & $\begin{array}{l}\text { Tuberculinés } \\
\text { et contrôlés }\end{array}$ & $\begin{array}{c}\text { Négatifs aux deux } \\
\text { tuberculines }\end{array}$ & $\begin{array}{l}\text { Positifs à la } \\
\text { tuberculine } \\
\text { humano-bovine }\end{array}$ & $\begin{array}{l}\text { Positifs à la } \\
\text { tuberculine } \\
\text { aviaire }\end{array}$ \\
\hline Groupe I & 157 & $\begin{array}{c}148 \\
(94,26 \mathrm{p} \cdot 100)\end{array}$ & $\left(0,64^{1} \mathrm{p} \cdot 100\right)$ & $\left(5,10^{8} \mathrm{p} \cdot 100\right)$ \\
\hline Groupe II & 669 & $\begin{array}{c}634 \\
(94,77 \mathrm{p} \cdot 100)\end{array}$ & $\frac{15}{(2,24 \mathrm{p}, 100)}$ & $\frac{20}{(2,99 \mathrm{p}, 100)}$ \\
\hline Groupe IJI & 813 & $\begin{array}{c}761 \\
(93,60 \mathrm{p} \cdot 100)\end{array}$ & $\begin{array}{c}25 \\
(3,08 \text { p. 100) }\end{array}$ & $(3,32$ p. 100) \\
\hline Groupe IV & 793 & $\begin{array}{l}726 \\
(91,55 \mathrm{p} \cdot 100)\end{array}$ & $(3,53 \mathrm{p} \cdot 100)$ & $(4,92 \mathrm{p}, 100)$ \\
\hline Total & 2.432 & $\begin{array}{l}2.269 \\
(93,30 \mathrm{p}, 100)\end{array}$ & $\begin{array}{c}69 \\
(2,84 \mathrm{p}, 100)\end{array}$ & $\begin{array}{c}94 \\
(3,86 \mathrm{p} \cdot 100)\end{array}$ \\
\hline
\end{tabular}


viennent transhumer des animaux en provenance de régions fortement infectées de ce pays: Moptı, Ségou, Macina (REGNOULT 1963). L'étude comparative des résultats des tuberculınations dans ces 2 secteurs montre qu'il existe une différence significative en ce qui concerne la tuberculine humano-bovine : 2,13 p. 100 de réactions positives à Dédougou et 4,65 p. 100 à Nouna (chi $2=10,46$ pour un degré de liberté et $P<0,001)$. Ces résultats sont reportés dans le tableau 5 . kuy dans le secteur de Nouna : 14 animaux positifs (soit 10,44 p. 100) sur 134.

Bien que de fréquence moindre que dans la région de Dori, la tuberculose bovine pose un problème dans cette région particulièrement exposée à la contagion par les animaux transhumants.

\subsubsection{Région de Boundiali.}

3,09 p. 100 des animaux tuberculınés ont réagi positivement à la tuberculine humano-bovine et

TABLEAU 5

Rêsultats des tuberculinations dans les secteurs de Dédougou et de Nouna (région de Dédougou)

\begin{tabular}{|c|c|c|c|c|c|c|c|}
\hline \multirow{2}{*}{$\begin{array}{c}\text { Secteurs } \\
\text { prospectês } \\
\text { dans la } \\
\text { Iégion } \\
\text { de Dédougou }\end{array}$} & \multirow{2}{*}{$\begin{array}{l}\text { Nombre d'animaux } \\
\text { tuberculinés et } \\
\text { contrôlés }\end{array}$} & \multicolumn{3}{|c|}{$\begin{array}{l}\text { Animaux positifs à la tuberculine } \\
\text { humano-bovine }\end{array}$} & \multicolumn{3}{|c|}{$\begin{array}{c}\text { Animaux positifs à la tuberculine } \\
\text { aviaire }\end{array}$} \\
\hline & & Nombre & Pourcentage & $\begin{array}{l}\text { Intervalle de } \\
\text { confiance } \\
\text { avec risque } \\
5 \text { p. } 100\end{array}$ & Nombre & Pourcentage & $\begin{array}{l}\text { Intervalle de } \\
\text { confiance } \\
\text { avec risque } \\
5 \text { p. } 100\end{array}$ \\
\hline DEDOUGOU & 1.765 & 38 & 2,13 & $1,46-2,80$ & 75 & 4,25 & $3,29-5,21$ \\
\hline NOUNA & 667 & 31 & 4,65 & $3,02-6,28$ & 19 & 2,85 & $1,56-4,14$ \\
\hline
\end{tabular}

Par contre le test statistique ne montre pas de différence significative pour la tuberculine aviaire (chi $2=2,47$ pour un degré de liberté). Comme dans la région de Dorı, on constate des variations importantes d'un village à l'autre (village de Bladi dans le secteur de Dédougou : aucun animal positif sur 148 ; village de Soumo-
3,45 p. 100 à la tuberculıne avıaire. Ces résultats ne diffèrent pas sensiblement de ceux obtenus dans la région de Dédougou. Les conditions écologıques de ces 2 régions, sans être semblables, diffèrent cependant moins entre elles qu'avec celles de la région de Dori.

Les résultats par groupe d'âge sont indiqués

TABLEAU 6

Rêsultats par groupe d'âge et par tuberculine pour la régıon de Boundiali

\begin{tabular}{|c|c|c|c|c|}
\hline \multirow[b]{2}{*}{ Groupes d'âge } & \multicolumn{4}{|c|}{ Nombre d'animaux } \\
\hline & $\begin{array}{l}\text { Tuberculinés } \\
\text { et contrôlês }\end{array}$ & $\begin{array}{c}\text { Négatifs aux deux } \\
\text { tuberculines }\end{array}$ & $\begin{array}{l}\text { Positifs à la } \\
\text { tuberculine } \\
\text { humano-bovine }\end{array}$ & $\begin{array}{l}\text { Positifs à la } \\
\text { tuberculine } \\
\text { aviaire }\end{array}$ \\
\hline Groupe I & 367 & $(90,19 \mathrm{p}, 100)$ & $(3,54 \mathrm{p} \cdot 100)$ & $\left(6,27^{23} \mathrm{p} \cdot 100\right)$ \\
\hline Groupe II & 409 & $\begin{array}{c}396 \\
(96,82 \mathrm{p} \cdot 100)\end{array}$ & $\left(0,25^{1} \mathrm{p} .100\right)$ & $\left(2,93^{12} \mathrm{p} \cdot 100\right)$ \\
\hline Groupe III & 455 & $\begin{array}{l}421 \\
(92,53 \mathrm{p}, 100)\end{array}$ & $(4,39 \mathrm{p} \cdot 100)$ & $(3,08 \stackrel{14}{p} \cdot 100)$ \\
\hline Groupe IV & 452 & $(9+.03 \mathrm{p} \cdot 100)$ & $(3,98 p \cdot 100)$ & $(1,99 \stackrel{9}{\mathrm{p} \cdot 100)}$ \\
\hline Tota1 & 1.683 & $\begin{array}{l}1.573 \\
(93.46 \mathrm{p} .100)\end{array}$ & $\begin{array}{c}52 \\
(3,09 \text { p. 100) }\end{array}$ & $(3,45 \mathrm{p} \cdot 100)$ \\
\hline
\end{tabular}


dans le tableau 6. L'étude statistique de ces résultats montre qu'une différence significative existe entre les différents groupes d'âge tant pour la tuberculine humano-bovine (chi $2=$ 14,65 pour 3 degrés de liberté et $0,01<P<0,02$ ) que pour la tuberculıne aviaire (chi $2=11,87$ pour 3 degrés de liberté et $0,01<\mathrm{P}<0,02$ ). Comme dans la région de Dori ce sont les animaux appartenant au groupe Il qui présentent le taux d'infection le moins élevé du point de vue tuberculose, tandis qu'en ce qui concerne la tuberculine aviaire, ce sont les animaux les plus jeunes qui ont présenté le pourcentage le plus élevé de réactions positives.

Comme dans les autres régions également, on constate des variations importantes d'un village à l'autre en ce qui concerne la tuberculine humano-bovine (villages de Sisséplé et M'bia : aucune réaction positive ; village de Nondara : 11 p. 100 de réactions positives).

La tuberculose bovine dans cette région revêt donc une importance sensiblement égale à celle reconnue dans la région de Dédougou et, comme cette dernière, la région de Boundiali est également exposée à la contagion d'animaux transhumants ou en transit destinés à la consommation et provenant de régions contaminées.

\subsubsection{Région de Daloa-Gagnoa.}

Les résultats concernant cette région sont exposés dans le tableau 7. Quatre animaux seulement ont réagi à la tuberculine humano- bovine, soi† 0,65 p. 100 des animaux tuberculinés ef contrôlés. Ces 4 animaux appartiennent soit au groupe III, soit au groupe IV. Aucun animal n'a réagi à la tuberculine aviaire. L'incidence de la tuberculose sur le chéptel de cette région peut donc être tenue pour néglıgeable. Toutefois, les animaux importés pour la consommation, ainsi que ceux en transit, constituent une menace permanente pour le cheptel local. II importe donc qu'une surveillance stricte soit mise en œuvre à leur égard afin d'éviter le contact avec le cheptel local, pour lequel des moyens importants sont mis en cuvre afin d'en favoriser le développement.

\subsubsection{Région de Sassandra.}

Les résultats concernant cette région sont exposés dans le tableau 8. La tuberculose bovine semble ne pas exister dans cette région puisqu'aucun animal n'a réagi à la tuberculine humano-bovine. Par contre nous avons noté 1,56 p. 100 de réactions positives vis-à-vis de la tuberculıne aviare. Comme dans les autres régions les réactions positives intéressent surtout les animaux jeunes.

\section{DONNÉES STATISTIQUES DES ABATTOIRS}

Ces données qui concernent les différents abattoirs des régions prospectées figurent dans. le tableau 9.

TABLEAU 7

Résultats par groupe d'âge et par tuberculine pour la région de Daloa-Gagnoa

\begin{tabular}{|c|c|c|c|c|}
\hline \multirow[b]{2}{*}{ Groupes d'âge } & \multicolumn{4}{|c|}{ Nonbre d'animaux } \\
\hline & $\begin{array}{l}\text { Tuberculinĕs } \\
\text { et contrôlës }\end{array}$ & $\begin{array}{c}\text { Negatifs aux deux } \\
\text { tuberculines }\end{array}$ & $\begin{array}{l}\text { Positifs à la } \\
\text { tuberculine } \\
\text { humano-bovine }\end{array}$ & $\begin{array}{l}\text { Positifs à la } \\
\text { tuberculine } \\
\text { aviaire }\end{array}$ \\
\hline Groupe I & 92 & $\left(100^{92}\right.$ p. 100$)$ & 0 & 0 \\
\hline Groupe II & 142 & $(100$ p. 100$)$ & 0 & 0 \\
\hline Groupe III & 222 & $\begin{array}{c}219 \\
(98.65 \text { p. 100) }\end{array}$ & $\left(1,35^{3} \mathrm{p} \cdot 100\right)$ & 0 \\
\hline Groupe IV & 157 & $\begin{array}{c}156 \\
(99,36 \mathrm{p} .100)\end{array}$ & $\left(0,64^{\mathrm{I}} \mathrm{p} \cdot 100\right)$ & 0 \\
\hline Total & 613 & $\begin{array}{c}609 \\
(99,35 \mathrm{p} \cdot 100)\end{array}$ & $\left(0,65^{4} p \cdot 100\right)$ & 0 \\
\hline
\end{tabular}


TABLEAU 8

Résultats par groupe d'âge et par tuberculine pour la rêgion de Sassandra

\begin{tabular}{|c|c|c|c|c|}
\hline \multirow{2}{*}{ Groupes d'âge } & \multicolumn{4}{|c|}{ Nombre d'animaux } \\
\hline & $\begin{array}{l}\text { Tuberculinés } \\
\text { et contrôlés }\end{array}$ & $\begin{array}{c}\text { Nëgatifs aux deux } \\
\text { tuberculines }\end{array}$ & $\begin{array}{l}\text { Positifs à la } \\
\text { tuberculine } \\
\text { humano-bovine }\end{array}$ & $\begin{array}{l}\text { Positifs à 1a } \\
\text { tuberculine } \\
\text { aviaire }\end{array}$ \\
\hline Groupe I & 74 & $\begin{array}{c}71 \\
\langle 95,95 \mathrm{p} \cdot 100\rangle\end{array}$ & 0 & $(4,05 \mathrm{p}, 100)$ \\
\hline Groupe II & 136 & $\begin{array}{l}131 \\
(96,32 \mathrm{p} \cdot 100)\end{array}$ & 0 & $\stackrel{5}{(3,68 \text { p. } 100)}$ \\
\hline Groupe III & 187 & $\begin{array}{c}186 \\
(99,47 \mathrm{p} \cdot 100)\end{array}$ & 0 & $\frac{1}{(0,53 \mathrm{p} \cdot 100)}$ \\
\hline Groupe IV & 180 & $\begin{array}{c}180 \\
(100 \mathrm{p} \cdot 100)\end{array}$ & 0 &, 0 \\
\hline Total & 577 & $\begin{array}{l}568 \\
(98,44 \text { p. } 100)\end{array}$ & 0 & $\stackrel{9}{(1,56 \mathrm{p} \cdot 100)}$ \\
\hline
\end{tabular}

TÁBLEAU 9

Nombre d'animaux abattus et nombre $d$ 'animaux reconnus tubcrculeux à 1 'inspection dans les différents abattoirs des rêgions prospectées

\begin{tabular}{|c|c|c|c|c|c|c|}
\hline \multirow{2}{*}{ Régions prospectées } & \multirow{2}{*}{ Abattoirs } & \multirow{2}{*}{ Années } & \multicolumn{2}{|c|}{ Nombre de bovins } & \multicolumn{2}{|c|}{ Forme de tuberculose } \\
\hline & & & Contrôlès & Tuberculeux & Généralisēe & Localisêz \\
\hline DORI (Haute-Vol ta) & Dori & $1963-1966$ & 2.741 & $\begin{array}{c}229 \\
(8,35 \mathrm{p}, 100)\end{array}$ & 27 & 202 \\
\hline \multirow[t]{3}{*}{$\begin{array}{l}\text { DEDOUGOU } \\
\text { (Haute-Volta) }\end{array}$} & Dëdoutgou & $1959-1966$ & 6.190 & $(6,64 \mathrm{p} \cdot 100)$ & 120 & 291 \\
\hline & Nouna & i $959-1966$ & 6.455 & $\begin{array}{c}803 \\
(12,44 p \cdot 100)\end{array}$ & 107 & 696 \\
\hline & Total & $1959-1966$ & 12.645 & $\begin{array}{c}1.214 \\
(9,66 \mathrm{p}, 100)\end{array}$ & 227 & 987 \\
\hline $\begin{array}{l}\text { BOUNDIALI } \\
\text { (Côte d'Ivoire) }\end{array}$ & Boundiali & $1965-1967$ & 3.796 & $(1,71$ p.100) & 8 & 57 \\
\hline $\begin{array}{l}\text { DALOA-GAGNOA } \\
\text { (Côte d'Ivoire) }\end{array}$ & Daloa-Gagnoa & $1964-1966$ & 21.432 & $\begin{array}{c}452 \\
(2,11 \mathrm{p}, 100) \\
\end{array}$ & 3 & 449 \\
\hline $\begin{array}{l}\text { SASSANDRA } \\
\text { (Côte d'Ivoire) }\end{array}$ & Sassandra & $1960-1967$ & 2.917 & $(0,55 \mathrm{p} \cdot 100)$ & non précisée & non prëcisêx \\
\hline
\end{tabular}

En ce qui concerne l'abattoir de Dori, nous constatons que le pourcentage d'animaux reconnus tuberculeux à l'abattage correspond presque exactement au pourcentage d'animaux appartenant au groupe d'âge IV (c'est-à-dire âgés de plus de 5 ans) ayant réagi positivement à la tuberculine humano-bovine. Aux abattoirs de Dédougou et de Nouna les pourcentages d'ani- maux fuberculeux sont supérieurs à ceux des bovins ayant réagi positivement à la tuberculıne humano-bovine. Certaınes années d'allleurs, le pourcentage d'animaux iuberculeux à l'abattoir de Nouna a été très supérıeur au chiffre moyen indiqué. C'est aınsi qu'en 1965, 23,55 p. 100 des animaux abattus ont présenté des lésions tuberculeuses à l'inspection. Les différences observées 
dans cette région entre les données statistiques des abattoirs et les résultats de l'enquête tuberculinique s'expliquent par le fait, que dans les deux abattors considérés, un pourcentage variable, mais parfois considérable des animaux abattus, ne sont pas originaires de la région mais proviennent précisément des zones fortement infectées dont nous avons parlé précédemment.

Dans la région de Boundiali au contraire, le pourcentage d'animaux reconnus tuberculeux aux abattoirs est nettement inférieur aux résultats de l'enquête tuberculinique. Nous pensons que les données statistiques concernant les saisies pour tuberculose à cet abattoir sont sujettes à caution. En effet, une partie des animaux abattus sont des zébus provenant souvent de régions contaminées.

Aux abattoirs de Daloa et Gagnoa la fréquence de la tuberculose est plus grande que ne le lassserait supposer les résultats de l'enquête tuberculinique. Cette différence s'explique aisément par le fait que les animaux abattus sont pour la plupart étrangers à la région prospectée.

Enfin, en ce qui concerne les chiffres de Sassandra, ceux-ci nous semblent très sujets à caution. En effet, pendant les 10 jours que nous avons passés à Sassandra même, au cours de notre enquête, nous avons eu l'occasion d'observer 2 cas de tuberculose bovine alors que pour toute l'année 1967, 2 cas seulement de tuberculose ont été identifiés sur 432 animaux contrôlés qui sont pour la grande majorité des bovins d'Importation.

Nous constatons donc en conclusion qu'il y a concordance entre les tests tuberculınıques et les chiffres observés aux abattoirs dans la mesure où les animaux abattus proviennent de la région prospectée. Dans le cas contraıre, qui est fréquent, les chiffres observés aux abattorrs sont évidemment fonction de l'origine des animaux abattus.

\section{DISCUSSION DES RÉSULTATS}

\subsection{Tuberculine humano-bovine.}

Les résultats de l'enquête tuberculinique montrent que des différences sıgnificatives existent d'une part entre les régions observées et d'autre part en fonction de l'âge des anımaux, le taux d'infection augmentant en général avec l'âge. Du point de vue régional, les régions les plus touchées, du point de vue tuberculose bovine sont celles où l'élevage est le plus important, quand i! n'est pas la seule ressource, comme c'est le cas dans les régions sahéliennes.

Dans ces régions le problème de la tuberculose bovine se pose donc sous le triple aspect vétérinaire, économique et santé publique.

\section{Aspect vétérnarre.}

La contagion de la tuberculose en élevage extensif est démontrée. Dans les régions infectées, les varıatıons de l'endémicité peuvent être grandes d'un village à l'autre. Certains villages peuvent être indemnes de tuberculose, alors que d'autres au contralre sont fortement infectés. A l'intérıeur d'un même village contaminé, on observe des variations également importantes d'un troupeau d̀ l'autre. Mais si, dans un tel village, les troupeaux sont plus ou moins atteints, ils sont néanmains toujours atteints. II ne s'agit que de variations quantitatives.

\section{Aspect économique.}

Les taux de 5 à 6 p. 100 d'animaux positifs constatés dans certaines régions à forte densité de cheptel doivent être tenus pour alarmants vu précisément l'importance du cheptel dans les régions concernées.

Aspect santé publique.

Les résultats des tuberculınatıons étudiés en fonction de l'âge ont montré que la tuberculose était la plus fréquente parmı les anımaux appartenant au groupe IV c'est-à-dire âgés de plus de 5 ans. Or, dans ce groupe, les femelles sont en forte majorité comme nous l'a montré notre enquête. Car, contrairement à une opinion répandue, mais qui semble erronée, nous n'avons que rarement rencontré des animaux âgés de sexe mâle. Or, dans les régıons sahélıennes en particulier, les populations trouvent la source essentielle de leurs protéines animales dans la consommation du lait de leur cheptel, et, ce latt est consommé très souvent à l'état cru. Ceci explique le taux particulièrement élevé des réactions positives observées chez les jeunes enfants et montre l'incidence certaine de la tuberculose bovine sur la population humaine 
de ces régions (voir commentaires dans la publication consacrée aux résultaìs de l'enquête humaine - ALBERT et al. 1969).

\subsection{Tuberculine aviaire.}

Ce sont dans les régions de Dédougou et de Boundiali que les pourcentages les plus élevés de réactıons positives ont été observés. Le nombre de réactions positives à cette tuberculine semble en rapport avec l'importance de l'aviculture dans la région prospectée. C'est aınsı que dans la région de Dori où le pourcentage de réactions positives est inférieur à 1 p. 100, les volailles sont très peu nombreuses et parfois même totalement absentes dans certaıns villages. Selon les villages, le taux de réactions positives est allé de 0 p. 100 à 2,5 p. 100 seulement. Dans les régıons de Dédougou et Boundıali, l'élevage des volailles est largement répandu dans la plupart des villages. Dans ces deux régıons aucun village n'a été indemne et les taux les plus élevés ont été respectivement de 11,2 p. 100 à Boundiali et de 15,8 p. 100 à Dédougou. Les volailles sont d'ailleurs plus nombreuses en général dans cette dernière région. A Daloa et Gagnoa, aucune réaction positive n'a été observée. A Sassandra où le taux moyen est très faible $(1,56 \mathrm{p}, 100)$ nous avons constaté des différences importantes d'un troupeau à l'autre. C'est ainsi qu'd̀ l'IRHO (Institut de recherches sur les huiles et les oléagineux), le premier troupeau tuberculiné comprenalt 268 animaux et a présenté 7 réactions positives tandis que sur les 124 animaux du second troupeau aucune réaction positive n'a été notée. Or, la ferme où se trouve le premier troupeau comprend de nombreuses volailles de race locale alors que celle où se trouve le deuxième troupeau n'a seulement que quelques volailles de race importée.

Enfin, si on étudie les résultats en fonction de l'âge, on constate que, dans toutes les régions, les taux les plus élevés de réactions positives sont rencontrés chez les anımaux les plus jeunes, le pourcentage de réactions positives tendant à décroître ensuite. On peut supposer que les très jeunes anımaux qui sont gardés au village dans la journée, pendant que le troupeau va paître en brousse, sont plus en contact avec les volaılles. Plus tard, lorsque cessera le contact avec l'agent contaminant, l'organisme se libérera de son infection en même temps que disparaîtra, au bout d'un certain délai, la sensibilisation (LUCAS 1967).

\subsection{Possibilité de réactions croisées avec Nocar- dia Forcinica, agent du farcin.}

On sait qu'il existe des liens antigéniques étroits entre le bacille tuberculeux et l'agent du farcin des bovidés. Nocardia Farcinica est notamment capable de faire apparaître une allergie à la tuberculine et des animaux atteints de farcin seront donc susceptıbles de donner une réponse faussement positive à une injection intradermique de tuberculine.

Mais, dans les différentes régions que nous avons prospectées au cours de nos enquêtes, le farcin n'a jamais été signalé jusqu'à présent, ni en clınıque $\mathrm{n}$ I en inspection des viandes. L'attention des agents responsables de cetie inspection dans les abattoirs avait cependant été atłırée sur la confusion possible entre les lésions dues à la fuberculose et celles imputables au farcin.

Cette absence du farcin dans les régions intéressées a été confirmée par la mise en culture de plus de 600 prélèvements d'origine animale au Laboratoire de la Tuberculose du Centre Muraz, dont les résultats font l'objet d'une cutre publication (GIDEL et al. 1969) et pour lesquels aucune souche de Nocardia Farcinica n'a été isolée.

\section{7. ÉTUDE D'UNE CORRÉLATION ENTRE LES TESTS TUBERCULINIQUES CHEZ L'HOMME ET CHEZ L'ANIMAL}

Rappelons tout d'abord que l'enquête humaine a été menée parallèlement à l'enquête animale et a donc intéressé les mêmes villages.

Schématiquement cette enquête a été conduite de la façon suivante :

Dans chaque village désigné par le tirage au sort, la population était divisée en 5 groupes d'âge :

- 0 à 18 mois (groupe $O$ ),

- 18 mois à 5 ans (groupe A),

- 6 à 12 ans (groupe B),

- 13 à 25 ans (groupe C),

- plus de 25 ans (groupe D). 
Dans chacun de ces groupes d'âge, 25 sujets furent sélectionnés par tirage au sort. Toutefois, l'étude des sujets âgés de 0 à 18 mois présenta de nombreuses difficultés et dut finalement être abandonnée.

L'étude des variations des résultats des tesłs tuberculiniques en fonction de l'âge et de la région permet les commentaires suivants, que l'on peut résumer ainsi :

Chez les enfants de 18 mois à 5 ans (groupe A), I'étude statistıque des ıntradermoréactıons montre qu'il existe une différence hautement significative en fonction de la région étudiée : chi $2=47,881$ pour 4 degrés de liberté et $P<0,001$. Sassandra présente le taux de réponses positives le plus faible ( 3,0 p. 100 pour 266 sujets testés) et Dori le taux le plus élevé (13,7 p. 100 pour 451 sujets testés). $\mathrm{Si}$, dans cette dernıère région, on prend les différents villages séparément, on constate une très grande hétérogénéité dans la répartition des enfants présentant une réaction d'hypersensibilité retardée à la tuberculıne. Les taux obtenus pour les 20 villages testés ont été les suivants :

$0,0-0,0-0,0-4,0-4,0-5,0-, 8,5-9,5-10,0-12,0$ $-12,0-13,0-14,0-16,0-19,0-27,0-29,0-29,5$ $30,0-33,0-$

Or, lorsqu'on compare les résultats de l'enquête humaine et de l'enquête animale, on constate que les villages où le taux de réactivité est le plus élevé pour chacune de ces enquêtes sont les mêmes. Ces variations suivant les villages donnent à penser qu'une des explications les plus logiques de ce phénomène est la consommation de lait cru provenant de bovins malades par les jeunes enfants des villages où le taux de réactions positives à la tuberculine est élevé.

Chez les enfants de 6 à 12 ans, des constatations à peu près semblables peuvent être faites, tandis que chez les sujets des groupes $C$ et $D$, on observe un phénomène assez paradoxal: alors que les taux de positivité sont pratiquement les mêmes pour les régions de Dédougou, Boundiali, Daloa et Sassandra (43,3 p. 100 à 45,2 p. 100 de réactions positives pour les sujets de 12 à 25 ans ; 59,4 p. 100 à 66,0 p. 100 pour les sujets de plus de 25 ans), la région de Dori présente des taux très inférieurs : 33,4 p. 100 et 45,5 p. 100 respectıvement. Ce fait est d'autant plus étonnant, que c'est précisément dans cette zone sahélıenne que l'on trouvait une réactivité importante à la tuberculine chez les enfants. D'cutre part lorsque, dans cette région, on reprend les villages individuellement comme nous l'avions fait pour les sujets de 18 mols à 5 ans, on ne constate plus de variations importantes.

La seule hypothèse que l'on pusse émettre et qui contient peut-être quelque réalité est la suivante : lorsque l'on considère les statistiques des abattoirs de la région, on ne peut qu'être frappé par la croissance très nette du taux d'animaux tuberculeux depuis une dizaine d'années. On pourrait donc admettre que les sujets nés avant cette période ont eu moins de "chance» d'être en contact avec un bacille tuberculeux au cours de leur jeune âge et que, d'autre part, le bacille tuberculeux, pénétrant par voie digestive chez un adulte ou un adolescent a bequcoup plus de difficultés à faıre apparaître une allergle chez ce type de sujets que chez l'enfant. Tout cecl se situant évidemment dans une région où la contamination se fait essentiellement par voie digestive. D'autre part, on peut également penser que si cette contamination est essentiellement digestive, les modes d'extérıorisation et de transmission de l'agent pathogène seront moins favorables et, par conséquent. l'atteınte des sujets réceptifs sera moins aisée que dans les régions où la contamination se fera principalement par voie cérienne.

\section{CONCLUSION}

Ces enquêtes par tests tuberculiniques ont confirmé les résultats de l'étude bibliographique faite par REGNOULT en 1963 et basée sur les documents officiels des différents Etats, en particulier les registres d'inspection des abattoirs. L'importance de la tuberculose bovine est très variable en Afrique Occıdentale selon les régions, mais pose un problème inquiétant dans certaines d'entre elles. II se trouve en effet que les plus atteintes sont celles où l'élevage est la ressource économique majeure. De plus, dans ces mêmes régions, la consommation de lait cru par les populations constitue la source essentielle de 
leurs protéines anımales. Malheureusement, du fait même des conditions partıculières d'élevage dans ces régions, du nomadisme des populations et de leur dispersion, les mesures à prendre pour enrayer l'extension de l'affection ne peuvent être que difficiles et onéreuses. Par contre, dans les zones à faible densité de cheptel, qui sont précisément les moins atteintes, mals où des moyens importants sont mis en ceuvre pour le développement de l'élevage, il importe que des mesures adéquates soient prises pour éviter la contamination de ce cheptel. Une surveillance strıcte doit être mise en œuvre afin d'éviter le contact avec les animaux en transit qui peuvent être originaires de régions infectées. Une tuberculınation du cheptel devrait être pratıquée tous les ans ou tous les deux ans afin de dépister et d'éliminer les anımaux éventuellement réagıssants. Tout anımal d'orıgıne étrangère introduit dans ces zones, devrait être soumıs à une tuberculination préalable en même temps d'cilleurs qu'à une prise de sang pour le dépistage de la brucellose et de la péripneumonie. Enfin, pour pallier l'incidence éventuelle de la tuberculose bovine sur la population humaine par la consommation du lait, il suffircit de mettre en œuvre un moyen sımple : la stérilisation du laıt par l'ébullition. La diffusion et l'application de cette mesure d'éducation sanitaire seront toutefois très difficiles à obtenir en zone sahélienne.

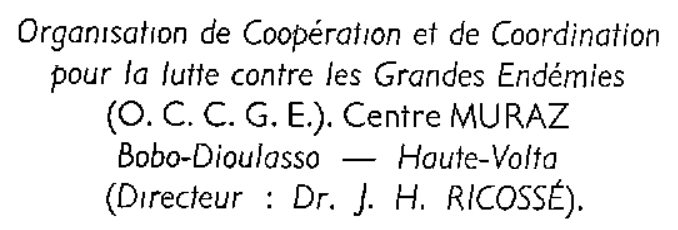

\section{REMERCIEMENTS}

Nous tenons à exprimer nos vifs remerciements à :

- Messieurs les Directeurs de l'Elevage de Côte d'lvoire et de Haute-Volta pour le concours qu'ils nous ont apporté en matıère de personnel ef de matériel.

- Messieurs les Commandants de Cercle, Préfets et Sous-Préfets des régions prospectées pour l'accueil qu'ils nous ont réservé.

- Messieurs les Chefs de Circonscription et Secteurs d'Elevage intéressés aınsi qu'à leur personnel pour l'alde précieuse qu'ils nous ont accordée et grâce à laquelle nous avons pu mener à bien notre travail sur le terrain.

- Monsieur le Docteur MENARD pour les conseils qu'il nous a donnés en matière de statistique.

\title{
SUMMARY
}

\author{
Survey on bovine tuberculosis by means of fuberculinic tests in different \\ regions of West Africa (Upper Volta and Ivory Coast). \\ Results and general considerations
}

In 1967 and 1968, the zoonosis and luberculosis subdivisions of Muraz Center in Bobo-Dioulasso, have carried out 5 jointed surveys on the epidemiology of human and bovine tuberculosis in West Africa. These surveys have been carried out by means of tuberculinic tests and surveys in different climatic zones of Upper-Volta and Ivary Coast, from the north lafifude $1^{\circ}$ (sahelian zone) to the north latitude $4^{\circ}$ (coasial zone).

The authors explain in this paper the results of these surveys with regard to the bovine fuberculosis. 8,132 cattle were submitted to the tuberculin test in the whole; each one with two simultaneous tuberculinations in two different parts by using human-bovine and avian tuberculins.

By the statistical analysis of results, significative differences are observed on the one hand between the prospected regions and on the other hand, in a same region, according to the age of animals submitted to the tuberculin test. Then, the comparative study of results of human and animal surveys in sahelıan region shows the incidence of bovine fuberculosis in the human people of these regions, parlicularly in the young children. 


\section{RESUMEN}

Encuesta sobre la tuberculosis bovina mediante pruebas tuberculinicas en diferentes regiones de Africa occidental (Alta Volta y Costa de Marfil). Resultados y consideraciones generales

En 1967 y 1968 , las subsecciones tuberculosis y zoonosis del Centro Muraz en Bobo-Dioulasso efectuaron 5 encuestas sobre la epidemiologia de la tuberculosis humana $y$ de la tuberculosis bovina en Africa del Oeste. Se efectuaron dichas encuestas mediante pruebas tuberculınicas y estadística aleatoria en varias zonas climáticas de Alta-Volta y de Costa de Marfil, desde el decimoquinto grado de latitud narle (zona saheliana) hasta el cuarto grado de latitud norte (zona costera).

Los autores exponen los resultados de dichas encuestas en lo concerniente a la tuberculosis de los bovinos. Se tuberculinizaron 8.132 bovinos en total. Se efectuó una doble tuberculinización en dos partes diferentes de cada animal con tuberculina humano-bovina y tuberculına aviar.

Según el analisis estadístico de los resultados, se observan diferencias significativas entre las regıones observadas $y$, en una misma región, según la edad de los animales tuberculinizados. Por fin, el estudio comparativo de los resultados de las encuestas humanas y animales en región saheliana muestra la incidencia de la tuberculosis bovina sobre la población humana de dichas regiones, partıcularmente en los pequeños niños.

\section{BIBLIOGRAPHIE}

AUBREVILLE (A.), - Flore forestière soudanoguinéenne. Soc, d'Edit. géog. Marit. et col., 1950, Paris, 523 Pp.

AUBREVILLE (A.), DUVIGNEAU (P.), HOYLES (A. C.), KEAY (R. W. J.), MENDONCA (F. A.) et PICHISERMOLLI (R. E. G.). Carte de la végétation de l'Afrique. London, Oxford University Press, 1958.

GORET (P.), SAURAT (P.) et LAUTITE (R.). Les divers types de bacilles tuberculeux et leur pouvoir pathogène pour l'homme. Interdépendance des tuberculoses humaines et animales. Rec. Méd. Vét., 1958, 134, 689714.

GORET (P.) et TOMA (B.). - Vers l'éradication de la tuberculose bovine. Rec. Méd. Vét., 1967, $143(7), 619-636$.

JOUBERT (L.) et OUDAR (J.). - Intertransmissibilité et prophylaxie des tuberculoses humaines ef animales, Le problème actuel des mycobactérioses atypiques. Goz. Méd. France, 1966, 3603-3616.

LEFEVRE (M.) et GATEFF (C.). - Mise au point d'une méthode de sondage lors d'une enquête tuberculinique en milieu rural africain. Méd. Trop., 1966, 26/3, 235-248.
LUCAS (A.). - Les erreurs par excès-risque de deuxième ordre ou des statisticiens au cours des fuberculinations. Bull. Assoc. Franç. Vét. Microb., 1968, 3, 53-56.

LUCAS (A.) et GAYOT (G.). - - Procédés actuels de dépistage de la tuberculose bovine. Cahiers Techniques du CNERNA, 1967, Paris.

OUDAR (J.), JOUBERT (L.), VIALLIER (J.), CAILLERE (F.) et GORET (P.). — Les mycobactéries chez les animaux. Leurs éventuelles transmissions à l'homme. Rev. Path. Comp., 1966, 66, 477-491.

PERPEZAT (A.), MARIAT (F.), DESTOMBES (P.) et THOME (M.). - Importance du farcin chez le zébu du Tchad. Bull. Soc. Path. Exot., 1963, 56, 375-383.

PLACIDI (L.) et ROUSSEL (A.). - Les fuberculoses humaines ef la tuberculose bovine. Rev. Path. Comp., 1964, 763, 527-532 ef 764, 7-11.

REGNOULT (M. G.). - La fuberculose animale dans les territoires Ouest-Africains d'expression française. Rev. Poth. Gén. et Phys. Clin., 1963, 573, 1093-1115.

SCHWARZ (D.). - Méthodes statistiques d̀ l'usage des médecins ef des biologistes. Editions Flammarion, 1963, Paris. 
THIBAULT (Ph.). - La tuberculose humaine d'origine bovine. Lo Presse Médicole, 1967. 75/26, 1344.

ALBERT (j. P.), GIDEL (R.) et RETIF (M.). Contribution à l'étude de l'épidémiologie de la tuberculose humaine en Afrique Occidentale. Résultats de 5 enquêtes effectuées au moyen de tests fuberculiniques et par sondage aléatoire dans différentes zones climatiques de Côte-d'lvoire et de HauteVolta à paraître in « Revue de Tuberculose et Pneumologie $\gg, 1970$.

GIDEL (R.), ALBERT (J. P.), LEFEVRE (M.), MENARD (M.) et RETIF (M.). - Les mycobactéries d'origine animale isolées au Centre Muraz de 1965 à 1968 . Techniques d'isolement ef d'identification. Résultats A paraître in Rev. Elev. Méd. Vét. Pays trop., 1969. 